\title{
INSULINA INALÁVEL: UMA ROTA TERAPÊUTICA SEGURA?
}

\section{INHALABLE INSULIN: A SAFE THERAPEUTIC ROUTE?}

\author{
Marina Assermann ${ }^{1}$, Paulo Ricardo Heinen ${ }^{2}$, Elaine De Oliveira Zanini ${ }^{3}$ \\ ${ }^{1}$ Acadêmico do curso de Medicina do Centro Universitário FAG - Cascavel-PR. ${ }^{2}$ Doutor em \\ Bioquímica pela USP, acadêmico do curso de Medicina FAG. ${ }^{3}$ Mestre em Desenvolvimento Regional \\ e Agronegócio pela Universidade Estadual do Oeste do Paraná. Docente do curso de Medicina do \\ Centro Universitário FAG - Cascavel. \\ *Autor Correspondente: ezanini@fag.edu.br, https://orcid.org/0000-0002-5098-7857
}

DOI: $10.35984 / f j h . v 2 i 1.170$

\section{RESUMO}

O diabetes mellitus é uma doença causada pela deficiência relativa ou absoluta do hormônio insulina, manifestando complicações macrovasculares e microvasculares em decorrência da hiperglicemia. Desde o primeiro uso de insulina subcutânea novas rotas foram pesquisadas tendo destaque a via pulmonar que é menos invasiva e mais cômoda. Diante disso, este trabalho levanta algumas considerações sobre a segurança clínica da insulina inalável Afrezza $\AA$, recentemente aprovada para comercialização no Brasil. Para isso, foi realizado estudo de revisão da literatura relacionado à insulina inalável, mediante busca eletrônica de artigos indexados na base de dados online SCIELO. Concisamente, os trabalhos relataram que a absorção da insulina por via pulmonar permite um início de ação mais rápido e uma duração de ação mais curta em relação aos análogos de insulina subcutânea. Os efeitos adversos mais comuns associados ao produto Afrezza ${ }^{\circledR}$ foram tosse, hipoglicemia, irritação da garganta e episódios de broncoespasmo agudo em pacientes asmáticos. Ainda assim, a insulinoterapia por via inalável representa um importante avanço para a reposição de insulina exógena, especialmente por dispensar o uso de agulhas. Contudo, o uso da insulina Afrezzaß por pacientes com doença pulmonar prévia deve ser ponderado, particularmente pelo possível risco associado a incidência de tumores no trato respiratório.

Palavras-chave: Insulina, Diabetes Mellitus, Afrezza ${ }^{\circledR}$.

\begin{abstract}
Diabetes mellitus is a disease caused by the relative or absolute deficiency of the hormone insulin, manifesting macrovascular and microvascular complications due to hyperglycemia. Since the first use of subcutaneous insulin, new routes have been researched, especially the less invasive and more comfortable pulmonary route. Given this, this paper raises some considerations about the clinical safety of Afrezza® inhaled insulin, recently approved for commercialization in Brazil. For this, a literature review study was performed related to inhalable insulin, using the search for indexed electronic articles in the SCIELO database. Concisely, work on pulmonary insulin absorption allows for faster onset of action and a shorter duration of action compared to subcutaneous insulin analogues. The most common adverse effects associated with Afrezza® were: hypoglycaemia, throat irritation and acute bronchospasm in asthmatic patients. Still, inhaled insulin therapy represents an important advance for exogenous insulin replacement, especially as it does not require the use of needles. However, the use of Afrezza® insulin by patients with prior lung disease should be considered, particularly because of the possible risk associated with the incidence of respiratory tract tumors.
\end{abstract}

Keywords: Insulin, Diabetes Mellitus, Afrezza ${ }^{\circledR}$. 


\section{INTRODUÇÃO}

O diabetes mellitus (DM) é uma doença metabólica crônica causada pela deficiência relativa ou absoluta do hormônio anabólico insulina. Sendo a insulina produzida pelo pâncreas com a função principal de regular os níveis de glicose no sangue, o DM manifesta-se fundamentalmente pela hiperglicemia, apresentando, por consequência, importantes complicações macrovasculares e microvasculares (FUCHS; WANNMACHER; FERREIRA, 2004). Em nível macrovascular, destaca-se o desenvolvimento de cardiopatia isquêmica, doença cerebrovascular e doença vascular periférica, que estão frequentemente associadas à morbimortalidade decorrente do diabetes mellitus. Já as complicações microvasculares são caracterizadas por retinopatia, nefropatia e neuropatias, que constituem as causas mais comuns de cegueira irreversível, doença renal crônica e amputações não traumáticas de membros inferiores, respectivamente (TSCHIEDEL, 2014; SANTOS et al., 2015).

Desde o primeiro uso terapêutico da insulina por Banting e Best, em 1921, modelos alternativos de administração de insulina têm sido pesquisados, incluindo sistemas de administração transdérmica, ocular, oral, bucal, nasal, retal, vaginal e uterina. No entanto, esses métodos historicamente falharam como sistemas de entrega de insulina, principalmente devido à baixa biodisponibilidade. A biodisponibilidade oral da insulina, por exemplo, é consideravelmente limitada, sobretudo, devido a sua intensa metabolização pré-sistêmica (CAVAIOLA; EDELMAN, 2014). Por tal motivo, o tecido subcutâneo ainda é a rota de administração mais utilizada para a reposição exógena de insulina em pacientes diabéticos (SHAH et al., 2016; GRADEL; PORSGAARD; LYKKESFELDT, 2018).

O sucesso da insulinoterapia, em grande parte, depende das propriedades farmacocinéticas e farmacodinâmicas da insulina e da utilização de técnicas corretas de aplicação. Embora a insulinoterapia tenha sido bem estabelecida como um agente eficaz para diminuir os níveis de hemoglobina glicosilada ( $\mathrm{HbA1c}$ ), muitos pacientes, especialmente os DM2, são muitas vezes relutantes em iniciar a terapia com insulina (WOUDENBERG et al., 2012). Os potenciais determinantes para essa resistência psicológica incluem dor antecipada, ansiedade, hipoglicemia, inconveniência e preocupação com o ganho de peso (CAVAIOLA; EDELMAN, 2014). Além disso, outra complicação associada à terapia insulínica relaciona-se com a ocorrência de lipodistrofia local, uma vez que o efeito anabólico direto da insulina pode provocar tanto a perda de tecido subcutâneo quanto o aumento de gordura localizada, isto porque, como esses locais tornam-se relativamente indolores, os pacientes tendem a injetar na mesma área repetidamente, em vez de mudar para um novo local (KADIYALA; WALTON; SATHYAPALAN, 2014).

Uma alternativa recentemente desenvolvida, não invasiva, eficaz e mais cômoda, é a insulina inalável. Seguindo os passos da agência reguladora de alimentos e medicamentos dos Estados Unidos (FDA, na sigla em inglês), a Agência Nacional de Vigilância Sanitária (ANVISA), em junho de 2019, aprovou a comercialização do produto Afrezza® no Brasil, que é uma insulina de ação rápida inalada por via oral para tratar diabetes em adultos. Dessa forma, o presente trabalho tem como objetivo lançar algumas considerações para fins de reflexão sobre a segurança clínica da insulina sob a forma farmacêutica inalável. 


\section{METODOLOGIA}

A análise realizada nesta investigação pauta-se em um estudo de revisão da literatura relacionada à insulina inalável. A pesquisa foi operacionalizada por meio da busca eletrônica de artigos indexados na base de dados online Scientific Eletronic Library Online (SciELO), utilizando-se os seguintes descritores: diabete mellitus, insulina inalável e câncer de pulmão. Os critérios de inclusão para o estudo foram: artigos em idioma português, espanhol e inglês, originais ou de revisão, publicados no período de 2000 a novembro de 2019, com textos disponíveis na íntegra abordando a temática acerca do tema em estudo. Foram excluídos: manuais, resumos de eventos, relatos de experiência e publicações não disponíveis na íntegra.

\section{RESULTADOS E DISCUSSÃO}

\subsection{Insulina inalável}

Dentre as alternativas de administração de insulina investigados até o momento, a administração por via pulmonar mostrou-se a mais promissora. Estudos têm demonstrado que a insulina inalável apresenta um ponderável aumento de biodisponibilidade, na ordem de 4 a 40 vezes, quando comparada as formulações retal, bucal e ocular. Esse resultado muito provavelmente se deve a extensa vascularização pulmonar. Estima-se que os pulmões possuam aproximadamente 500 milhões de alvéolos, abrangendo uma área superficial de $50-140 \mathrm{~m}^{2}$ em indivíduos adultos. Some-se a isto, a barreira alvéolo-capilar, por sua espessura extremamente fina e delgada, garante a rápida e eficiente absorção da insulina, embora apenas $59 \%$ da dose inalada chegue até os pulmões (CAVAIOLA; EDELMAN, 2014).

O tamanho das partículas apresenta-se como um dos fatores mais importantes na administração de insulina por via pulmonar. Pesquisas indicam uma absorção mais eficiente com o uso de partículas entre 1 e $3 \mu \mathrm{m}$ de diâmetro, visto que partículas maiores são depositadas nas vias aéreas superiores ou engolidas (CAVAIOLA; EDELMAN, 2014). Em relação aos análogos de insulina subcutânea, a absorção da insulina via pulmonar permite um início de ação mais rápido e uma duração de ação mais curta, demonstrando um perfil fisiológico semelhante ao da insulina endógena em pacientes não diabéticos (PITTAS; WESTCOTT; BALK, 2015).

\subsection{Insulina Exubera ${ }^{\circledR}$}

A insulina Exubera $\AA^{\circledR}$, uma forma inalável de insulina de ação rápida desenvolvida pela Pfizer, tornou-se o primeiro produto de insulina inalada a ser aprovada pela FDA, em 2006, mas as más vendas do produto levaram a Pfizer a interromper a sua comercialização alguns meses depois, em 2007 (MOHANTY; DAS, 2017). Além disso, devido a sua via de administração, foram relatados muitos efeitos adversos no aparelho respiratório, incluindo um importante aumento no risco de infecção respiratória, tosse, faringite e rinite. Alguns estudos também ilustraram um declínio estatisticamente maior na função pulmonar em comparação ao grupo placebo (OLECK; KASSAM; GOLDMAN, 2016). Pode ser que, como um hormônio anabólico, a insulina simplesmente aumente o tônus da musculatura lisa local e torne os pulmões mais "rígidos". Ainda assim, a atividade promotora de crescimento da insulina continua sendo a maior preocupação para a sua aplicação a longo prazo nos pulmões, uma vez que pode promover câncer em fumantes atuais ou ex-fumantes pesados (HEINEMANN, 2018). 
A atividade promotora de crescimento da insulina decorre de sua fraca interação com o receptor do fator de crescimento semelhante à insulina 1 (GLP-1), com 1/150 da potência do GLP-1. Em animais, nenhum estudo tem relatado qualquer efeito substancial sobre a proliferação celular em áreas alveolares ou brônquicas mediante uso de insulina inalável. Por outro lado, em humanos, os ensaios clínicos evidenciam uma maior incidência de câncer de pulmão, cerca de 0,13 para 100 pacientes/ano tratados com a insulina Exubera $\AA$, em comparação com 0,03 para os pacientes não expostos a essa medicação. Todavia, concluiu-se que haviam poucos casos para determinar a causalidade dos eventos e que todos os pacientes que desenvolveram câncer de pulmão possuíam um histórico prévio de tabagismo (CAVAIOLA; EDELMAN, 2014; OLECK; KASSAM; GOLDMAN, 2016).

\subsection{Insulina Afrezza®}

No decorrer dos últimos anos, por meio da tecnologia tecnosfera, uma nova insulina inalável para adultos foi aprovada pela FDA, em junho de 2014 (FANTASIA, 2015; MOHANTY; DAS, 2017). A tecnosfera é um sistema de administração de medicamentos composto por micropartículas de fumarildicetopiperazina (FDCP) que, em $\mathrm{pH}$ levemente ácido, formam microesferas de 2-5 $\mu \mathrm{m}$ por meio de ligações de hidrogênio. A insulina humana, assim como outros peptídeos e proteínas, podem ser eficientemente carreadas por essas microesferas (CAVAIOLA; EDELMAN, 2014; SARALA; BENGALORKAR; BHWANA, 2012; PIRES; CHACRA, 2008). Fornecida com um inalador pequeno, discreto e fácil de usar, a insulina tecnosfera Afrezza® é rapidamente absorvida em contato com a superfície pulmonar e, em contraste com a insulina Exubera® (8-9\%), possui um percentual residual no epitélio pulmonar de apenas 0,3\% após 12 horas (HEINEMANN, 2018; MOHANTY; DAS, 2017).

Nos Estados Unidos, a insulina Afrezza® vem sendo comercializada apenas nas doses de 4, 8 e 12 unidades. Isso significa que, ao contrário dos análogos de insulina regular subcutânea, disponíveis em doses de 1 unidade, a insulina inalável apresenta menor flexibilidade de dosagem, podendo exigir incrementos adicionais de insulina por via subcutânea para otimizar a cobertura da insulina prandial (HEINEMANN, 2018). Além disso, os pacientes que necessitam de insulina de ação prolongada, especialmente aqueles que possuem DM1, ainda precisam injetar rotineiramente suas doses basais. Portanto, o uso da insulina inalável reduz o número total de injeções administradas por dia, o que é atraente, mas não elimina completamente a necessidade das injeções subcutâneas (FANTASIA, 2015; OLECK, KASSAM; GOLDMAN, 2016).

\subsection{Farmacocinética e farmacodinâmica}

As propriedades farmacocinéticas e farmacodinâmicas da insulina Afrezza® foram pesquisadas por Rave et al. (2009), comparando o perfil de diferentes doses da insulina inalável Afrezza® $(25,50$ e 100 U) ao da insulina regular subcutânea (10 U). Constatou-se, por meio desse estudo, que o tempo médio para atingir a concentração sérica máxima foi alcançado aproximadamente duas horas mais cedo nos grupos expostos a insulina inalável (12-17 minutos) em relação ao grupo exposto a insulina subcutânea. No teste de biodisponibilidade, as doses de 25, 50 e $100 \mathrm{U}$ da insulina Afrezza ${ }^{\circledR}$ apresentaram biodisponibilidade relativa de $25 \%, 23 \%$ e $21 \%$, respectivamente. Além disso, o efeito biológico máximo, medido pela taxa de infusão de glicose, também ocorreu notavelmente mais cedo para as três doses do produto 
Afrezza ${ }^{\circledR}(42,50$ e 58 minutos) em comparação com insulina regular subcutânea (171 minutos).

Os estudos farmacodinâmicos da insulina Afrezza® demostraram um perfil de ação muito mais próximo ao fisiológico se comparado aos análogos de insulina subcutânea. A maior parte do efeito da insulina Afrezza $\AA^{\circledR}$ na redução dos níveis de glicose ocorreu durante as primeiras 3 horas $(71 \%)$, em contraste com a insulina regular subcutânea, que apresentou uma redução de apenas $27 \%$ em igual período (CAVAIOLA; EDELMAN, 2014). Além disso, nenhuma outra formulação de insulina demonstrou um início de ação igualmente rápido (PFÜTZNER; FORST, 2005). Para Rave et al. (2008), é possível que essas características se traduzam em um menor risco de hipoglicemia pós-prandial.

\subsection{Efeitos adversos e segurança}

Os efeitos adversos mais comuns associados a insulina Afrezza $\AA$ foram tosse, hipoglicemia e irritação da garganta (MOHANTY; DAS, 2017). Em um estudo clínico randomizado e aberto conduzido por Raskin et al. (2012), 27,8\% (257/923) dos pacientes que fizeram uso dessa medicação experimentaram tosse em comparação com apenas 4,4\% (42/949) dos pacientes que receberam o tratamento usual. A tosse relatada, predominantemente leve e improdutiva, ocorreu no primeiro mês após 0 início do tratamento e diminuiu ao longo do tempo. Embora a tosse não possa, de forma direta, causar problemas clínicos, é considerada um importante incômodo para a maioria dos pacientes (OLECK; KASSAM; GOLDMAN, 2016). Além disso, em decorrência do uso da insulina inalável, episódios de broncoespasmos agudos foram observados em 5 dos 17 indivíduos previamente diagnosticados com asma, já na primeira inalação, levando o FDA a exigir uma estratégia de mitigação de riscos e a inclusão de uma advertência em caixa sobre o risco aumentado de broncoespasmo agudo em pacientes com doença pulmonar obstrutiva crônica (OLECK; KASSAM; GOLDMAN, 2016; HEINEMANN, 2018).

Em relação ao risco de carcinogenicidade, mesmo que não haja qualquer evidência de risco de malignidade pulmonar pelo uso da insulina Afrezza ${ }^{\circledR}$ em animais, os resultados obtidos pelo uso desse produto em humanos, por sua vez, colocam em xeque a sua segurança (OLECK; KASSAM; GOLDMAN, 2016). Durante os ensaios clínicos, quatro casos de câncer de pulmão foram diagnosticados dentre os 2.750 pacientes expostos a insulina Afrezza®, sendo que, em dois destes casos, havia um histórico de tabagismo importante como fator de risco. No grupo controle, por outro lado, nenhum indivíduo foi diagnosticado com câncer de pulmão. De acordo com a empresa fabricante, esses dados são insuficientes e não definitivos, do ponto de vista estatístico, para determinar se a insulina Afrezza ${ }^{\circledR}$ está associada ao desenvolvimento de cânceres do trato respiratório (OLECK; KASSAM; GOLDMAN, 2016; FANTASIA, 2015).

No que se refere a segurança, o produto Afrezza $\AA$ é classificada na categoria $\mathrm{C}$ de risco na gravidez. Ainda que malformações fetais não tenham sido observadas em modelos animais, essa insulina inalável não foi testada em mulheres grávidas e, portanto, não deve ser usada mulheres que requerem reposição exógena de insulina durante a gravidez, a menos que o benefício potencial justifique o risco potencial para o feto. Acrescenta-se também que o uso dessa medicação é contraindicado em mulheres que estão amamentando, uma vez que tanto a insulina quanto as partículas do excipiente foram detectadas no leite materno durante os testes em animais. Sendo assim, é possível que o leite humano também contenha essas substâncias. No caso 
de menores de 18 anos, a contraindicação decorre da ausência de estudos com pacientes dessa faixa etária (MANKIND CORPORATION, 2014; FANTASIA, 2015).

\section{CONSIDERAÇÕES FINAIS}

A insulinoterapia por via inalável representa um importante avanço para a reposição de insulina exógena, especialmente para aqueles que apresentam hiperglicemia pós-prandial. $\mathrm{O}$ produto $A f r e z z a \circledR$, recentemente aprovado para comercialização no Brasil, possui um perfil de ação muito mais rápido do que os análogos de insulina administrados por via subcutânea. Além disso, por dispensar o uso de agulhas, espera-se que as barreiras psicológicas associadas à administração subcutânea de insulina sejam completamente superadas por meio dessa nova formulação. Contudo, o produto Afrezza® não foi aprovada para uso em gestantes, crianças e adolescentes, e o seu uso em pacientes com doença pulmonar prévia deve ser rigorosamente avaliado frente aos riscos e benefícios, particularmente pela inexistência de estudos definitivos sobre a sua associação com a incidência de tumores no trato respiratório

\section{REFERÊNCIAS}

FANTASIA, H. C. Inhaled insulin - a new delivery for an old drug. Nurs Womens Health, v. 19, n. 1, p. 67-70, 2015. https://doi.org/10.1111/1751-486X.12177

FINEBERG, S. E.; KAWABATA, T.; FINCO-KENT, D.; LIU, C.; KRASNER, A. Antibody response to inhaled insulin in patients with type 1 or type 2 diabetes. An analysis of initial phase II and III inhaled insulin (Exubera) trials and a two year extension trial. The Journal of Clinical Endocrinology and Metabolism, v. 90, n. 6, p. 3287-3294, 2005. https://doi.org/10.1210/jc.2004-2229

FUCHS, D.; WANNMACHER, L.; FERREIRA, M. B. C. Farmacologia clínica: fundamentos da terapêutica racional, $4^{\mathrm{a}}$. edição. Rio de janeiro: Guanabara Koogan, 2006.

CAVAIOLA, T. C; EDELMAN, S. Inhaled insulin: a breath of fresh air? A review of inhaled insulin. Clinical Therapeutics, v. 36, n. 8, p. 1275-1289, 2014. https://doi.org/10.1016/j.clinthera.2014.06.025

GRADEL, A. K. J.; PORSGAARD, T.; LYKKESFELDT, J. Factors affecting the absorption of subcutaneously administered insulin: effect on variability. Journal of Diabetes Research, v. 2018, p. 1-17, 2018. https://doi.org/10.1155/2018/1205121

HEINEMANN, L. Inhaled Insulin: Dead Horse or Rising Phoenix? Journal of Diabetes

Science and Technology, v. 12, n. 2, p. 239 -242, 2018. https://doi.org/10.1177/ 1932296817748231

KADIYALA, P.; WALTON, S.; SATHYAPALAN, T. Insulin induced lipodystrophy. The British Journal of Diabetes and Vascular Disease, v. 14, p. 131-133, 2014. http://dx.doi.org/10.15277/bjdvd.2014.036 
MANKIND CORPORATION. (2014). Highlights of prescribing information. Disponível em: www.mannkindcorp.com/Collateral/Documents/EnglishUS/Afrezza Prescribinglnform ation.pdf. Acesso em: 01 de nov. 2019.

MOHANTY, R. R.; DAS, S. Inhaled Insulin - Current Direction of Insulin Research. Journal of Clinical and Diagnostic Research, v. 11, n. 4, p. OE01-OE02, 2017. https://doi.org/10.7860/JCDR/2017/23626.9732

OLECK, J.; KASSAM, S.; GOLDMAN, D. J. Commentary: Why Was Inhaled Insulin a Failure in the Market? American Diabetes Association, v. 29, n. 3, p. 180-184, 2016. https://doi.org/10.2337/diaspect. 29.3.180

PFÜTZNER, A.; FORST, T. Pulmonary insulin delivery by means of the Technosphere drug carrier mechanism. Expert Opinion on Drug Delivery, v. 2, n. 6, p. 1097-1106, 2005. https://doi.org/10.1517/17425247.2.6.1097

PIRES, A. C.; CHACRA, A. R. A. Evolução da Insulinoterapia no Diabetes Melito Tipo 1. Arquivos Brasileiros de Endocrinologia e Metabolismo, v. 52, n. 2, p. 268-278, 2008. http://dx.doi.org/10.1590/S0004-27302008000200014

PITTAS, G. A.; WESTCOTT, P. G; BALK, M. E. Efficacy, safety, and patient acceptability of Technosphere inhaled insulin for people with diabetes: a systematic review and meta-analysis. Lancet Diabetes Endocrinoly, v. 3, n. 11, p. 886-894, 2015. http://dx.doi.org/10.1016/ S2213-8587(15)00280-6

RASKIN, P.; HELLER, S.; HONKA, M.; CHANG, P. -C.; BOSS, A. H.; RICHARDSON, P. C.; AMIN, N. Pulmonary function over 2 years in diabetic patients treated with prandial inhaled Technosphere Insulin or usual antidiabetes treatment: a randomized trial. Diabetes, Obesity and Metabolism, v. 14, n. 2, p. 163-173, 2012. http://dx.doi.org/10.1111/j.1463-1326.2011.01500.x

RAVE, K.; HEISE, T.; HEINEMANN, L.; BOSS, A. H. Inhaled Technosphere ${ }^{\circledR}$ Insulin in Comparison to Subcutaneous Regular Human Insulin: Time Action Profile and Variability in Subjects with Type 2 Diabetes. Journal of Diabetes Science and $\begin{array}{lllllll}\text { Technology, } & \text { v. } & \text { 2., } & \text { n. } & 2, & \text { p. } & 205-212,\end{array}$ http://dx.doi.org/10.1177/193229680800200206

RAVE, K.; POTOCKA, E.; BOSS, A. H.; MARINO, M.; COSTELLO, D.; CHEN, R. Pharmacokinetics and linear exposure of AFRESA compared with the subcutaneous injection of regular human insulin. Diabetes, Obesity and Metabolism, v. 11, p. 715720, 2009. http://dx.doi.org/10.1111/j.1463-1326.2009.01039.x

REIS, C. P. Novas alternativas terapêuticas para a administração oral da insulina. Revista lusófona de ciências e tecnologias em saúde, v. 5, n. 2, p. 174-205, 2008.

SANTOS, A. L.; CECÍLIO, H. P. M.; TESTON, E. F.; ARRUDA, G. O.; PETERNELLA, F. M. N.; MARCON, S. S. Microvascular complications in type 2 diabetes and 
associated factors: a telephone survey of self-reported morbidity. Ciência e Saúde Coletiva, v. 20, p. 761-770, 2015. https://doi.org/10.1590/1413$\underline{81232015203.12182014}$

SARALA, N.; BENGALORKAR, G.; BHWANA, K. Technosphere: new drug delivery system for inhaled insulin. Future Prescriber, v.13, n. 1, p. 14-16, 2012. https://doi.org/10.1002/fps.90

SHAH, R.; PATEL, M.; MAAHS, D.; SHAH, V. Insulin delivery methods: Past, present and future. International Journal of Pharmaceutical Investigation, v. 6, n. 1, p. 1-9, 2016. https://doi.org/10.4103/2230-973X.176456

TSCHIEDEL, B. Complicações crônicas do diabetes. Jornal Brasileiro de Medicina, v. 102, n. 5, p. 7-12, 2014.

WOUDENBERG, Y. J. C.; LUCAS, C.; LATOUR, C.; SCHOLTE OP REIMER, W. J. M. Acceptance of insulin therapy: a long shot? Psychological insulin resistance in primary care. Diabetic Medicine, v. 29, p. 796-802, 2012. https://doi.org/10.1111/j.1464-5491.2011.03552.x 